\title{
SUMMARY AND IMPORTANCE OF THE RESULTS INVOLVING THE DEFINITION OF $S$-CONVEXITY
}

\author{
Marcia Pinheiro \\ RGMIA \\ P.O. Box 12396 A'Beckett St \\ Melbourne, VIC, 8006, AUSTRALIA
}

\begin{abstract}
In this note, we try to summarize the results we have so far in terms of the definition of the $S$-convexity phenomenon, but we also try to explain in detail the relevance of those. For some of those results, we dare presenting graphical illustrations to make our point clearer. S-convexity came to us through the work of Prof. Dr. Dragomir (2001) and Prof. Dr. Dragomir claimed to have had contact with the concept through the hands of Hudzik and Maligranda, who, in their turn, mention Breckner and Orlicz as an inspiration. We are working in a professional way with the phenomenon since the year of 2001, and that was when we presented our first talk on the topic. In that talk, we introduced a conjecture about the shape of $S$-convexity. We have examined possible examples, we have worked with the definition and examples, and we then concluded that we needed to refine the definition by much if we wanted to still call the phenomenon an extensional phenomenon in what regards Convexity. We are now working on the fourth paper about the shape of $S$-convexity and trying to get both limiting lines (negative and non-negative functions) to be as similar as possible. It is a delicate labour to the side of Real Analysis, Vector Algebra, and even Calculus.
\end{abstract}

Key Words: analysis, convexity, $S$-convexity, $s$-convexity, geometry, shape

\section{Introduction}

In First Note on the Shape of $S$-convexity, we have presented more evidence on

Received: January 30, 2016

Published: March 2, 2016 (c) 2016 Academic Publications, Ltd.

url: www.acadpubl.eu 
our re-wording of the piece of definition of the $s$-convexity phenomenon that deals with non-negative real functions being of fundamental importance for Mathematics and we have proposed a geometric definition for the phenomenon.

In Second Note on the Shape of $S$-convexity, we have once more proved that the modifications to the definition of the $s$-convexity phenomenon proposed by us, this time for the negative share of the real functions, constitute a major step towards making the phenomenon be a proper extension of Convexity. We have also proposed a geometric definition for the negative case.

We hypothesized that our limiting curve for $s$-convexity, when the real function is negative, could be a bit bigger than the limiting curve for $s$-convexity when the real function is not negative in terms of length, what would mean that our lift could not be the same for both cases.

Our perimeters seemed to be close enough in dimension, however, the difference being noticed by the first decimal digit only and being less than 0.5 (considering our approximation for $p i$, our manual calculations, and the approximation for the perimeter via elliptical curve).

In Third Note on the Shape of $s$-convexity, we studied the perimeter of our limiting curve for the $s$-convexity phenomenon aiming equal perimeters for geometrically equivalent situations.

The limiting curve originates in the application of the definition of the phenomenon, so that, as a consequence, we proposed new refinements to the definition of the phenomenon at the end of it.

The conclusions, for our third note, were:

1) There might be exponents that are nicer than our chosen $\delta_{1}$ and $\delta_{2}$ and are still acceptable.

2) We have decided to deal with the $s$-convexity phenomenon as if it were an exclusively extensional concept for issues that have to do with practicality and accuracy (we now forbid $s$ to assume the value 1 in our definition).

3) We have extended the domain of the $s$-convex functions to $\Re$ because the definition should only bring necessary limitations, and we have found problems only with the image of the functions so far in what regards the current shape of the definition of the phenomenon we study, not the domain.

4) We have decided to swap the coefficients in our definition because $\lambda=0$ should bring $f(x)$ to life, not $f(x+\delta)$.

5) We have added the interval of definition of $s$ to our geometric definition to make it be independent from the analytical definition. 
6) Because of our new findings and decisions, we have produced a new update for our definition of the $s$-convexity phenomenon.

Update on our refinements of

the definitions proposed by Hudzik and Maligranda

\section{1) Analytical Definition}

We have two possibilities that far for each piece of the analytical definition of the phenomenon if we think of including the definition of Hudzik and Maligranda in Mathematics.

\section{A) Possibility 1}

Definition 1. A function $f: X \rightarrow \Re$, where $|f(x)|=f(x)$, is told to belong to $K_{\mathrm{s}}^{2}$ if the inequality

$$
f((1-\lambda) x+\lambda(x+\delta)) \leq(1-\lambda)^{\mathrm{s}} f(x)+\lambda^{\mathrm{s}} f(x+\delta)
$$

holds $\forall \lambda / \lambda \in[0,1] ; \forall x / x \in X ; s=s_{2} / 0<s_{2}<1 ; X / X \subseteq \Re \wedge X=[a, b]$; $\forall \delta / 0<\delta \leq(b-x)$.

Definition 2. A function $f: X \rightarrow \Re$, where $|f(x)|=-f(x)$, is told to belong to $K_{\mathrm{s}}^{2}$ if the inequality

$$
f((1-\lambda) x+\lambda(x+\delta)) \leq(1-\lambda)^{\log _{2}\left(\frac{1}{1-2^{-s}}\right)} f(x)+\lambda^{\log _{2}\left(\frac{1}{1-2^{-s}}\right)} f(x+\delta)
$$

holds $\forall \lambda / \lambda \in[0,1] ; \forall x / x \in X ; s=s_{2} / 0<s_{2}<1 ; X / X \subseteq \Re \wedge X=[a, b]$; $\forall \delta / 0<\delta \leq(b-x)$.

Remark 1. If the inequalities are obeyed in the reverse ${ }^{1}$ situation by $f$, then $f$ is said to be $s_{2}$-concave.

\footnotetext{
${ }^{1}$ Reverse here means ' $>$ ', not ' $\geq$ '.
} 


\section{B) Possibility 2}

Definition 3. A function $f: X \rightarrow \Re$, where $|f(x)|=f(x)$, is told to belong to $K_{\mathrm{s}}^{2}$ if the inequality

$$
f((1-\lambda) x+\lambda(x+\delta)) \leq(1-\lambda)^{\log _{2} \frac{1}{1-2^{-\frac{1}{s}}}} f(x)+\lambda^{\log _{2} \frac{1}{1-2^{-\frac{1}{s}}}} f(x+\delta)
$$

holds $\forall \lambda / \lambda \in[0,1] ; \forall x / x \in X ; s=s_{2} / 0<s_{2}<1 ; X / X \subseteq \Re \wedge X=[a, b]$; $\forall \delta / 0<\delta \leq(b-x)$.

Definition 4. A function $f: X \rightarrow \Re$, where $|f(x)|=-f(x)$, is told to belong to $K_{\mathrm{S}}^{2}$ if the inequality

$$
f((1-\lambda) x+\lambda(x+\delta)) \leq(1-\lambda)^{\frac{1}{s}} f(x)+\lambda^{\frac{1}{s}} f(x+\delta)
$$

holds $\forall \lambda / \lambda \in[0,1] ; \forall x / x \in X ; s=s_{2} / 0<s_{2}<1 ; X / X \subseteq \Re \wedge X=[a, b]$; $\forall \delta / 0<\delta \leq(b-x)$.

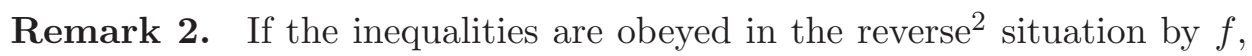
then $f$ is said to be $s_{2}$-concave.

\section{2) Geometric Definition}

We then have two possibilities for each piece of the geometric definition as well.

\section{A) Possibility 1}

Definition 5. A real function $f: X \rightarrow Y$, for which $|f(x)|=f(x)$, is called $s$-convex ${ }^{3}$ if and only if, for all choices $\left(x_{1} ; y_{1}\right)$ and $\left(x_{2} ; y_{2}\right)$, where $\left\{x_{1}, x_{2}\right\} \subset X,\left\{y_{1}, y_{2}\right\} \subset Y, Y=I m_{\mathrm{f}}$, and $x_{1} \neq x_{2}$, it happens that the line drawn between $\left(x_{1} ; y_{1}\right)$ and $\left(x_{2} ; y_{2}\right)$ by means of the expression $(1-\lambda)^{\mathrm{S}} y_{1}+\lambda^{\mathrm{S}} y_{2}$, where $\lambda \in[0,1]$, does not contain any point with height, measured against the vertical Cartesian axis, that is inferior to the height of its horizontal equivalent in the curve representing the ordered pairs of $f$ in the interval considered for the line in terms of distance from the origin of the Cartesian axis.

\footnotetext{
${ }^{2}$ See the previous footnote.

${ }^{3} s$ must be replaced, as needed, with a fixed constant located between 0 and 1 but different from 0 and 1 . For instance, if the chosen constant is 0.5 , then the function will be 0.5 -convex or $\frac{1}{2}$-convex and $s$ will be 0.5 in the expression that defines the limiting line.
} 
Definition 6. A real function $f: X \rightarrow Y$, for which $|f(x)|=-f(x)$, is called $s$-convex ${ }^{4}$ if and only if, for all choices $\left(x_{1} ; y_{1}\right)$ and $\left(x_{2} ; y_{2}\right)$, where $\left\{x_{1}, x_{2}\right\} \subset X,\left\{y_{1}, y_{2}\right\} \subset Y, Y=\operatorname{Im}_{\mathrm{f}}$, and $x_{1} \neq x_{2}$, it happens that the line drawn between $\left(x_{1} ; y_{1}\right)$ and $\left(x_{2} ; y_{2}\right)$ by means of the expression

$$
(1-\lambda)^{\log _{2}\left(\frac{1}{1-2^{-s}}\right)} y_{1}+\lambda^{\log _{2}\left(\frac{1}{1-2^{-s}}\right)} y_{2},
$$

where $\lambda \in[0,1]$, does not contain any point with height, measured against the vertical Cartesian axis, that is inferior to the height of its horizontal equivalent in the curve representing the ordered pairs of $f$ in the interval considered for the line in terms of distance from the origin of the Cartesian axis.

Remark 3. If all the points defining the function are located above the limiting line instead, then $f$ is called $s$-concave.

\section{B) Possibility 2}

Definition 7. A real function $f: X \rightarrow Y$, for which $|f(x)|=f(x)$, is called $s$-convex ${ }^{5}$ if and only if, for all choices $\left(x_{1} ; y_{1}\right)$ and $\left(x_{2} ; y_{2}\right)$, where $\left\{x_{1}, x_{2}\right\} \subset X,\left\{y_{1}, y_{2}\right\} \subset Y, Y=\operatorname{Im}_{\mathrm{f}}$, and $x_{1} \neq x_{2}$, it happens that the line drawn between $\left(x_{1} ; y_{1}\right)$ and $\left(x_{2} ; y_{2}\right)$ by means of the expression

$$
(1-\lambda)^{\log _{2}\left(\frac{1}{1-2^{-\frac{1}{s}}}\right)} y_{1}+\lambda^{\log _{2}\left(\frac{1}{1-2^{-\frac{1}{s}}}\right)} y_{2},
$$

where $\lambda \in[0,1]$, does not contain any point with height, measured against the vertical Cartesian axis, that is inferior to the height of its horizontal equivalent in the curve representing the ordered pairs of $f$ in the interval considered for the line in terms of distance from the origin of the Cartesian axis.

Definition 8. A real function $f: X \rightarrow Y$, for which $|f(x)|=-f(x)$, is called $s$-convex ${ }^{6}$ if and only if, for all choices $\left(x_{1} ; y_{1}\right)$ and $\left(x_{2} ; y_{2}\right)$, where $\left\{x_{1}, x_{2}\right\} \subset X,\left\{y_{1}, y_{2}\right\} \subset Y, Y=\operatorname{Im}_{\mathrm{f}}$, and $x_{1} \neq x_{2}$, it happens that the line drawn between $\left(x_{1} ; y_{1}\right)$ and $\left(x_{2} ; y_{2}\right)$ by means of the expression $(1-\lambda)^{\frac{1}{s}} y_{1}+\lambda^{\frac{1}{s}} y_{2}$, where $\lambda \in[0,1]$, does not contain any point with height, measured against the

\footnotetext{
${ }^{4}$ See the previous footnote.

${ }^{5}$ See the previous footnote.

${ }^{6}$ See the previous footnote.
} 
vertical Cartesian axis, that is inferior to the height of its horizontal equivalent in the curve representing the ordered pairs of $f$ in the interval considered for the line in terms of distance from the origin of the Cartesian axis.

Remark 4. If all the points defining the function are located above the limiting line instead, then $f$ is called $s$-concave.

\section{Development}

We should probably talk about how important our suggested modifications were for the concept and definition of the S-convexity phenomenon .

We start with the dimensions involved in the definition.

The original definition of the phenomenon was given by Hudzik and Maligranda according to Dragomir and it read [1]:

A function $f:[0, \infty) \rightarrow \Re$ is said to be $s$-convex in the first sense if

$$
f(a x+b y) \leq a^{\mathrm{s}} f(x)+b^{\mathrm{s}} f(y),
$$

$\forall x, y \in[0, \infty)$ and $\forall a, b \geq 0$ with $a^{\mathrm{S}}+b^{\mathrm{S}}=1,0<s \leq 1$.

A function $f:[0, \infty) \rightarrow \Re$ is said to be $s$-convex in the second sense if

$$
f(a x+b y) \leq a^{\mathrm{s}} f(x)+b^{\mathrm{s}} f(y),
$$

$\forall x, y \in[0, \infty)$ and $\forall a, b \geq 0$ with $a+b=1,0<s \leq 1$.

We then observed that we were aiming at graphs in $\Re^{2}$, and therefore we were drawing from $\Re$, but we were using two variables from the domain instead, what would have to mean $\Re^{2}$ in the domain.

Maple fails because we have a $3 D$ graph. Observe that the previous example brings plot $3 d$ and this one brings plot, which is the command for 2D graphs.

These plots are to show that, in the way it would be, after the introduction of the spacer, we still have three dimensions instead of two for our limiting line. Notwithstanding, we will be changing the wording of our definition once more in this paper to fix also this problem. We will say that for each $x$ we select from our domain, the inequality should be verified, so that we would be replacing $x$ with a constant on our next plot, what would finally give us two dimensions.

To disappear with one dimension, we introduced a constant, a spacer, as we said in the previous paragraph, and we called it $\delta$.

We also observed that we could not possibly be willing to allow for the person to get a situation in which $x=y$ because we then would not have the 
$>\operatorname{plot} 3 d\left(\left[x, a^{0.5} x+(1-a)^{0.5}(x+1)\right], a=0.1, x=0.1\right) ;$ with $($ plots $) ;$

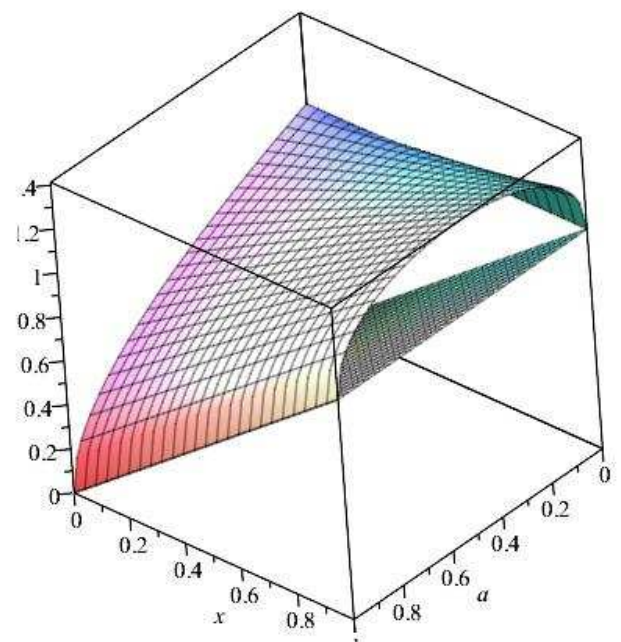

Figure 1: Maple Plot I

Hudzik and Maligranda: $x, y$, and $a$.

This graph: $x$ and $a$.

What we need is $a$ (only).

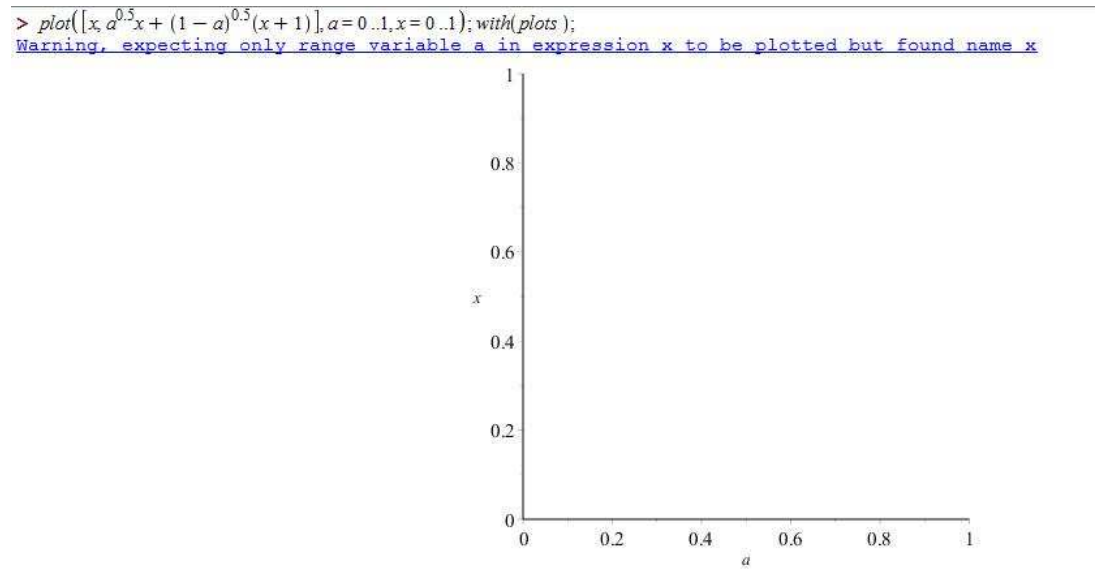

Figure 2: Maple Fails

geometric extension of the concept Convexity, but getting this extension could 
only be our main aim.

As a consequence of taking notice of the just-mentioned facts, we have proposed a refinement to the definition of the convexity phenomenon as well.

Once we chose our $x$, we would naturally choose a $y$ after it and a $y$ that is inside of the interval, and let's call it $I$, so that we can say that we have $x$ and $x+\delta$ where $\delta \neq 0$ and $\delta$ is between $x$ and the end of $I$.

We observed that $b$ depended on our choice of value for $a$, since $a^{\mathrm{s}}+b^{\mathrm{s}}=1$ or $a+b=1$. In this case, one of them could be called dependant variable, for it would be given in function of the other.

We also observed that convexity could use any domain in the reals, but Sconvexity, that far, was using only the non-negative part, what then eliminated the possibility of it being a proper extension of Convexity.

Upon examining the restriction from closer, we did not find any reason not to have the domain in the reals instead.

As a consequence of the just-mentioned findings, which we communicated to Academia also through [2], [3], and [4], we refined the definition of the phenomenon a bit more.

Upon studying the definitions for longer, we found counter-examples: Families of functions that would not fit inside of the concept $S$-convexity and yet were convex. The problem was that $S$-convexity lifted and bent the convexity line, so that the comparison signs and expressions could not fit both the case of the negative and the case of the positive convex functions. We then had to split the definition into two subdefinitions: one for when the modulus of the function equated the function and one for when the modulus of the function were equal to minus the function.

The original definition of S-convexity was, as we see on the paragraphs above this one, split into two other definitions: $s$-convex in the first sense and $s$-convex in the second sense. They were called $S_{1}-$ and $S_{2}-$ convex functions, respectively.

One of the senses could not be considered an extension of convexity, as we proved on [5], so that we had to drop it.

We were then seen talking about splitting only the second sense of $S$-convexity, the one that remained, $S_{2}$, into two subdefinitions. That is seen on [6].

Finally, we found out that, even with all the just-mentioned fixings, our split definition presented a mistake, which was a bit more rope to one of the subdefinitions than to the other one in what had to do with the limiting line: We needed to make sure that equivalent situations would return equivalent limiting lines, regardless of one being to the negative and the other being to the positive side. 
That is when we came up with another exponent, as we see in [4].

We also refined the only geometric definition we found for the convexity phenomenon as we proposed the first ever seen geometric definition of the $S$-convexity phenomenon. These results also appear on [4].

As the reader can see, $a$ and $b$ are interchangeable in the definition attributed to Hudzik and Maligranda. We, however, tied the position of the coefficients. We did this because otherwise the limiting line would not be acceptable. See the graphs:

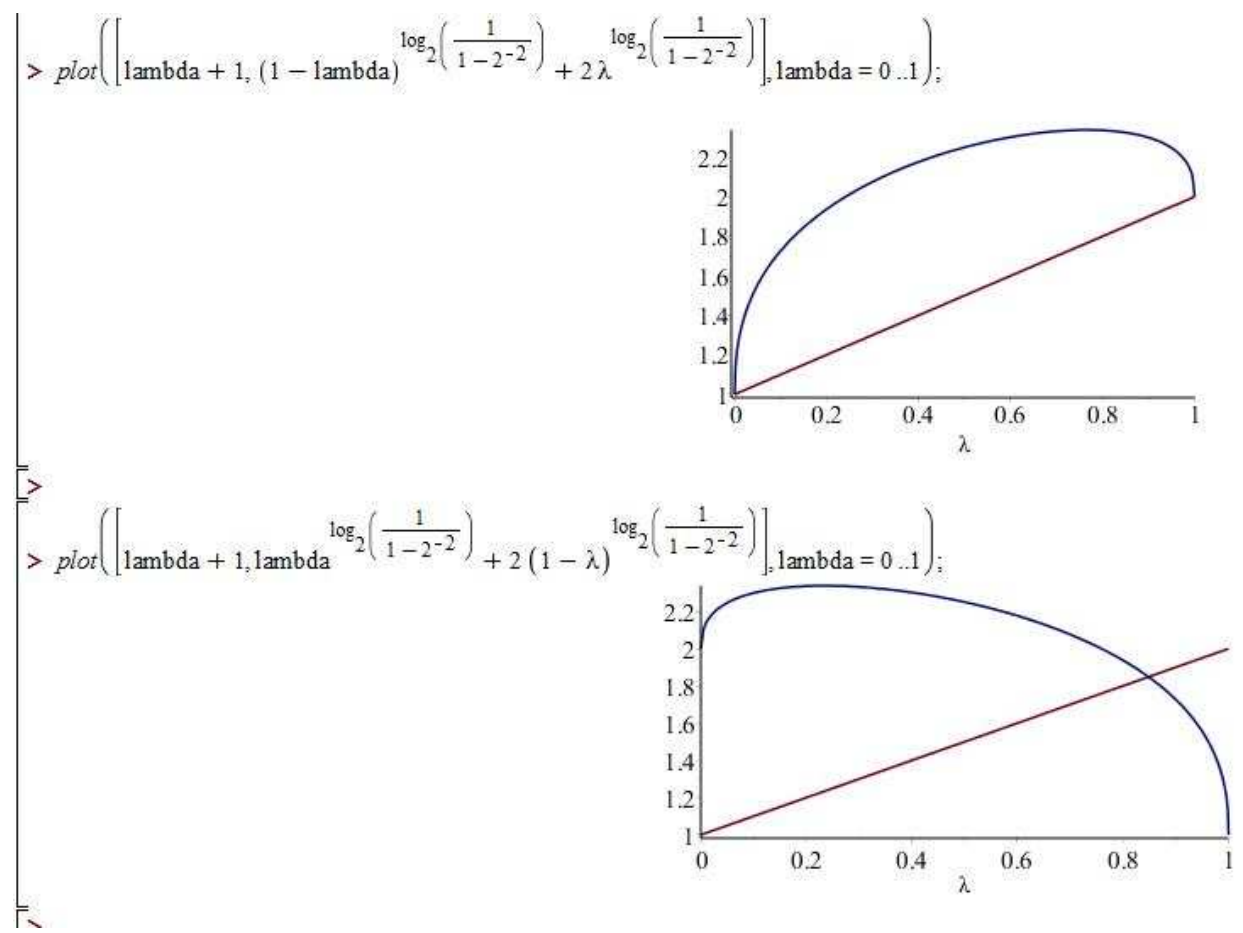

Figure 3: Coefficients, importance of the order

These are probably the most meaningful modifications that we have proposed to the definition of this phenomenon and we now need to tell the reader about our next step.

Having reached clarity and coherence in our definition, we now wonder about whether this is the best extension for the convexity phenomenon: Could we find something better, perhaps more analytical or more perfect?

The first idea that occurred to us was that we could have a piece of a circumference as a limiting line, so that we would achieve maximum analyticity. 
We could then choose to have the midpoint of the limiting line for convexity involved somehow in the determination of the center and the distance between that midpoint and the extremes as a radius. One of the problems with that is that we lose the variation of the $s$ and we then have something totally different. Another problem is that the expression will look totally different from that of the limiting line for convexity, and therefore will not allow us to easily compare them. There is also an issue with the concept extension: That would be perhaps a geometric extension, but not necessarily an analytical one. Besides, we then get an expression that seems to be independent of the function itself, for it depends solely on the extreme values picked from any chosen interval. With convexity, however, and also with the current concept of $S$-convexity, things depend on the value of the function at each stage of the comparison path. It perhaps stops being an extension and becomes simply an inequality if we introduce this change.

Any attempt to have the same geometric shape over all convexity limiting lines as a limiting line for $S$-convexity is then a vain attempt.

Even though we could probably have different exponents, it looks like our extension is now an excellent choice. When we studied the exponents, we concluded that only one of them could be nice (negative/non-negative) if we wanted to have equivalent situations returning equivalent shapes. That has been done on [4].

Our extension, as it is now, extends the concept of convexity both analytically and geometrically, so that it does look like an ideal choice.

We notice upon examining Figure 4 and Figure 5 (they both appear after this paragraph), that our limiting lines are not yet perfect, since, ideally, they would both have the same shape and dimensions (non-negative and negative case). Even though we put some effort into equating what we called the size of the rope, we have only equated the middle point match on the limiting line in terms of height, as one can tell by simply studying our developments in [4]. 


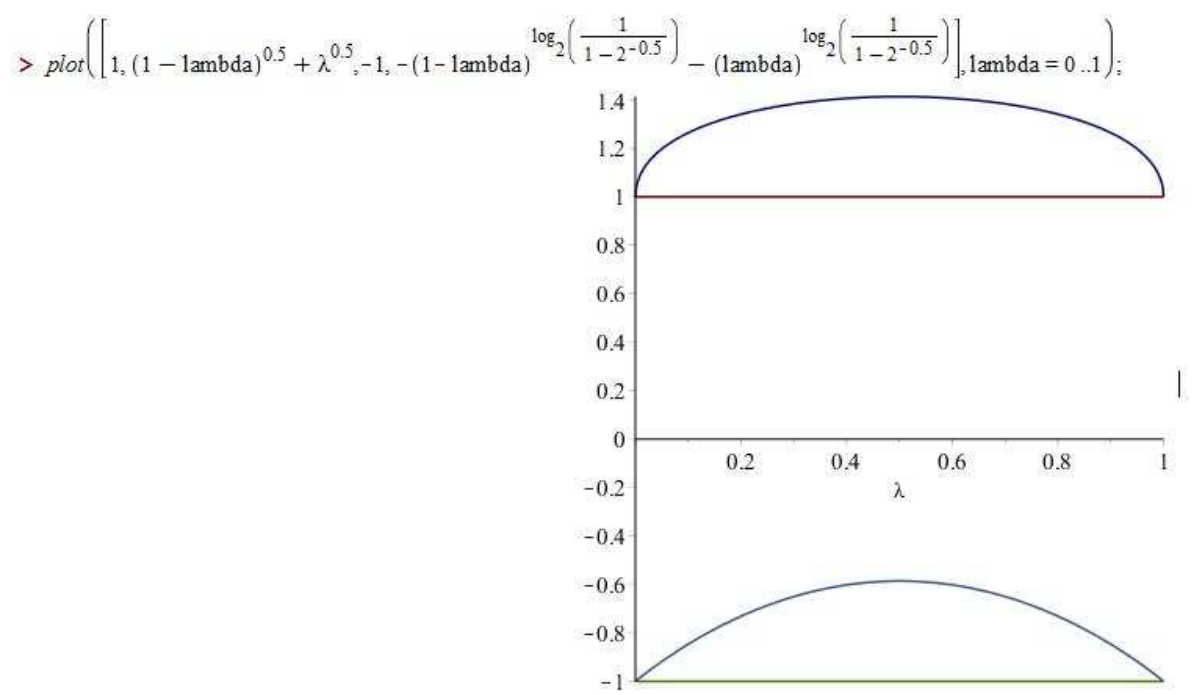

Figure 4: $S_{1}$

$>\operatorname{plot}\left(\left[-1,1,(1-\right.\right.$ lambda $\left.) \log _{2}\left(\frac{1}{1-2^{-\frac{1}{0.5}}}\right)+\lambda \log _{2}^{\log _{2}\left(\frac{1}{1-2^{-\frac{1}{0.5}}}\right)},-(1-\text { lambda })^{\frac{1}{0.5}}-1 \lambda^{\frac{1}{0.5}}\right]$, lambda $\left.=0 \ldots 1\right)$

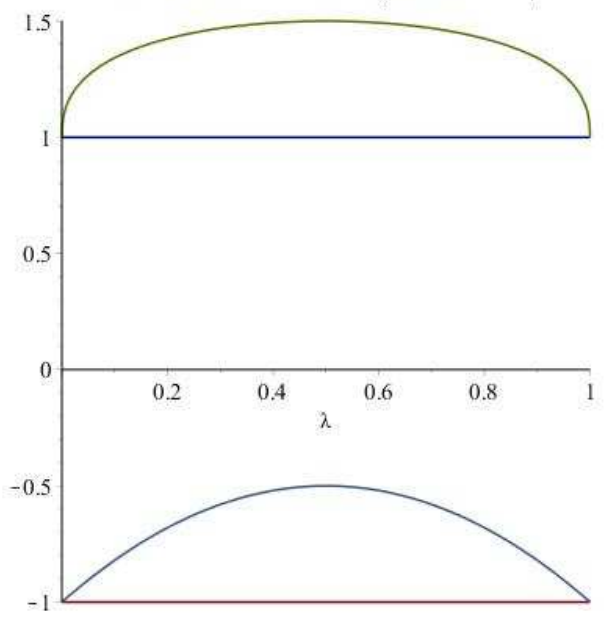

Figure 5: $S_{2}$ 


\section{Conclusion}

We have decided to keep the name $S_{1}$ and replace the previous class $K_{\mathrm{s}}^{1}$ with a new version of it, which would be one of our possible definitions, as for [4].

So far, we have:

1) Analytical Definition

$S_{1}$

Definition 1. A function $f: X \rightarrow \Re$, where $|f(x)|=f(x)$, is told to belong to $K_{\mathrm{s}}^{1}$ if, for each $x \in X$ we select, and for all of them, the inequality

$$
f((1-\lambda) x+\lambda(x+\delta)) \leq(1-\lambda)^{\mathrm{S}} f(x)+\lambda^{\mathrm{S}} f(x+\delta)
$$

holds $\forall \lambda / \lambda \in[0,1] ; s=s_{1} / 0<s_{1}<1 ; X / X \subseteq \Re \wedge X=[a, b] ; \forall \delta / 0<\delta \leq(b-x)$.

Definition 2. A function $f: X \rightarrow \Re$, where $|f(x)|=-f(x)$, is told to belong to $K_{\mathrm{S}}^{1}$ if, for each $x \in X$ we select, and for all of them, the inequality

$$
f((1-\lambda) x+\lambda(x+\delta)) \leq(1-\lambda)^{\log _{2}\left(\frac{1}{1-2^{-s}}\right)} f(x)+\lambda^{\log _{2}\left(\frac{1}{1-2^{-s}}\right)} f(x+\delta)
$$

holds $\forall \lambda / \lambda \in[0,1] ; s=s_{1} / 0<s_{1}<1 ; X / X \subseteq \Re \wedge X=[a, b] ; \forall \delta / 0<\delta \leq(b-x)$.

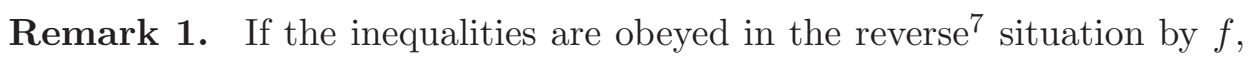
then $f$ is said to be $s_{1}$-concave.

$$
S_{2}
$$

Definition 3. A function $f: X \rightarrow \Re$, where $|f(x)|=f(x)$, is told to belong to $K_{\mathrm{s}}^{2}$ if, for each $x \in X$ we select, and for all of them, the inequality

$$
f((1-\lambda) x+\lambda(x+\delta)) \leq(1-\lambda)^{\log _{2} \frac{1}{1-2^{-\frac{1}{s}}}} f(x)+\lambda^{\log _{2} \frac{1}{1-2^{-\frac{1}{s}}}} f(x+\delta)
$$

holds $\forall \lambda / \lambda \in[0,1] ; s=s_{2} / 0<s_{2}<1 ; X / X \subseteq \Re \wedge X=[a, b] ; \forall \delta / 0<\delta \leq(b-x)$.

\footnotetext{
${ }^{7}$ Reverse here means ' $>$ ', not ' $\geq$ '.
} 
Definition 4. A function $f: X \rightarrow \Re$, where $|f(x)|=-f(x)$, is told to belong to $K_{\mathrm{s}}^{2}$ if, for each $x \in X$ we select, and for all of them, the inequality

$$
f((1-\lambda) x+\lambda(x+\delta)) \leq(1-\lambda)^{\frac{1}{s}} f(x)+\lambda^{\frac{1}{s}} f(x+\delta)
$$

holds $\forall \lambda / \lambda \in[0,1] ; s=s_{2} / 0<s_{2}<1 ; X / X \subseteq \Re \wedge X=[a, b] ; \forall \delta / 0<\delta \leq(b-x)$.

Remark 2. If the inequalities are obeyed in the reverse ${ }^{8}$ situation by $f$, then $f$ is said to be $s_{2}$-concave.

\section{2) Geometric Definition}

We then have two possibilities for each piece of the geometric definition as well.

\section{$S_{1}$}

Definition 5. A real function $f: X \rightarrow Y$, for which $|f(x)|=f(x)$, is called $s$-convex ${ }^{9}$ if and only if, for all choices $\left(x_{1} ; y_{1}\right)$ and $\left(x_{2} ; y_{2}\right)$, where $\left\{x_{1}, x_{2}\right\} \subset X,\left\{y_{1}, y_{2}\right\} \subset Y, Y=\operatorname{Im}_{\mathrm{f}}$, and $x_{1} \neq x_{2}$, it happens that the line drawn between $\left(x_{1} ; y_{1}\right)$ and $\left(x_{2} ; y_{2}\right)$ by means of the expression $(1-\lambda)^{\mathrm{s}} y_{1}+\lambda^{\mathrm{s}} y_{2}$, where $\lambda \in[0,1]$, does not contain any point with height, measured against the vertical Cartesian axis, that is inferior to the height of its horizontal equivalent in the curve representing the ordered pairs of $f$ in the interval considered for the line in terms of distance from the origin of the Cartesian axis.

Definition 6. A real function $f: X \rightarrow Y$, for which $|f(x)|=-f(x)$, is called $s$-convex ${ }^{10}$ if and only if, for all choices $\left(x_{1} ; y_{1}\right)$ and $\left(x_{2} ; y_{2}\right)$, where $\left\{x_{1}, x_{2}\right\} \subset X,\left\{y_{1}, y_{2}\right\} \subset Y, Y=\operatorname{Im}_{\mathrm{f}}$, and $x_{1} \neq x_{2}$, it happens that the line drawn between $\left(x_{1} ; y_{1}\right)$ and $\left(x_{2} ; y_{2}\right)$ by means of the expression

$$
(1-\lambda)^{\log _{2}\left(\frac{1}{1-2^{-s}}\right)} y_{1}+\lambda^{\log _{2}\left(\frac{1}{1-2^{-s}}\right)} y_{2},
$$

where $\lambda \in[0,1]$, does not contain any point with height, measured against the vertical Cartesian axis, that is inferior to the height of its horizontal equivalent

\footnotetext{
${ }^{8}$ See the previous footnote.

${ }^{9} s$ must be replaced, as needed, with a fixed constant located between 0 and 1 but different from 0 and 1 . For instance, if the chosen constant is 0.5 , then the function will be 0.5 -convex

or $\frac{1}{2}$-convex and $s$ will be 0.5 in the expression that defines the limiting line.
${ }^{10}$ See the previous footnote.
} 
in the curve representing the ordered pairs of $f$ in the interval considered for the line in terms of distance from the origin of the Cartesian axis.

Remark 3. If all the points defining the function are located above the limiting line instead, then $f$ is called $s$-concave.

\section{$S_{2}$}

Definition 7. A real function $f: X \rightarrow Y$, for which $|f(x)|=f(x)$, is called $s$-convex ${ }^{11}$ if and only if, for all choices $\left(x_{1} ; y_{1}\right)$ and $\left(x_{2} ; y_{2}\right)$, where $\left\{x_{1}, x_{2}\right\} \subset X,\left\{y_{1}, y_{2}\right\} \subset Y, Y=I m_{\mathrm{f}}$, and $x_{1} \neq x_{2}$, it happens that the line drawn between $\left(x_{1} ; y_{1}\right)$ and $\left(x_{2} ; y_{2}\right)$ by means of the expression

$$
(1-\lambda)^{\log _{2}\left(\frac{1}{1-2^{-\frac{1}{s}}}\right)} y_{1}+\lambda^{\log _{2}\left(\frac{1}{1-2^{-\frac{1}{s}}}\right)} y_{2}
$$

where $\lambda \in[0,1]$, does not contain any point with height, measured against the vertical Cartesian axis, that is inferior to the height of its horizontal equivalent in the curve representing the ordered pairs of $f$ in the interval considered for the line in terms of distance from the origin of the Cartesian axis.

Definition 8. A real function $f: X \rightarrow Y$, for which $|f(x)|=-f(x)$, is called $s$-convex ${ }^{12}$ if and only if, for all choices $\left(x_{1} ; y_{1}\right)$ and $\left(x_{2} ; y_{2}\right)$, where $\left\{x_{1}, x_{2}\right\} \subset X,\left\{y_{1}, y_{2}\right\} \subset Y, Y=I m_{\mathrm{f}}$, and $x_{1} \neq x_{2}$, it happens that the line drawn between $\left(x_{1} ; y_{1}\right)$ and $\left(x_{2} ; y_{2}\right)$ by means of the expression $(1-\lambda)^{\frac{1}{s}} y_{1}+\lambda^{\frac{1}{s}} y_{2}$, where $\lambda \in[0,1]$, does not contain any point with height, measured against the vertical Cartesian axis, that is inferior to the height of its horizontal equivalent in the curve representing the ordered pairs of $f$ in the interval considered for the line in terms of distance from the origin of the Cartesian axis.

Remark 4. If all the points defining the function are located above the limiting line instead, then $f$ is called $s$-concave.

We may come back to the subject shape in the near future to see if we can get even closer to our goal.

\footnotetext{
${ }^{11}$ See the previous footnote.

${ }^{12}$ See the previous footnote.
} 


\section{References}

[1] Hudzik, H., Maligranda, L. (1994). Some remarks on si-convex functions, Aequationes Mathematicae 48, 100-111.

[2] Pinheiro, M. R. (2012). Minima Domain Intervals and the S-Convexity, as well as the Convexity, Phenomenon, Advances in Pure Mathematics 2, 457-458.

[3] Pinheiro, M. R. (2004). Exploring the concept of S-Convexity, Proceedings of the WSEAS International Conference on Mathematics and Computers in Physics (MCF '04).

[4] Pinheiro, M. R. (2014). Third Note on the Shape of S-Convexity, International Journal of Pure and Applied Mathematics 93(5), 729-739.

[5] Pinheiro, M. R. (2015). Second Note on the Definition of $S_{1}$-convexity, Advances in Pure Mathematics 5, 127-130.

[6] Pinheiro, M. R. (2011). First Note on the Definition of $S_{2}$-convexity, Advances in Pure Mathematics 1, 1-2. 
